University of Nebraska - Lincoln

DigitalCommons@University of Nebraska - Lincoln

November 1987

\title{
Numerical methods for free-free radiative transition matrix elements
}

Bo Gao

University of Nebraska - Lincoln

Anthony F. Starace

University of Nebraska-Lincoln, astarace1@unl.edu

Follow this and additional works at: https://digitalcommons.unl.edu/physicsstarace

Part of the Physics Commons

Gao, Bo and Starace, Anthony F., "Numerical methods for free-free radiative transition matrix elements" (1987). Anthony F. Starace Publications. 87.

https://digitalcommons.unl.edu/physicsstarace/87

This Article is brought to you for free and open access by the Research Papers in Physics and Astronomy at DigitalCommons@University of Nebraska - Lincoln. It has been accepted for inclusion in Anthony F. Starace Publications by an authorized administrator of DigitalCommons@University of Nebraska - Lincoln. 


\title{
Numerical methods for free-free radiative transition matrix elements
}

\author{
Bo Gao and Anthony F. Starace \\ Department of Physics and Astronomy, The University of Nebraska, Lincoln, Nebraska 68588- \\ 0111
}

(Received 20 July 1987; accepted 19 August 1987)

Increasing interest in multiphoton absorption processes above the ionization threshold has led theorists to reexamine numerical techniques for calculating radiative transition matrix elements between states of a continuum electron moving at large radial distances in the field of an atom or an ion. Here it is shown that accurate free-free radial matrix elements may be obtained using the usual dipole length formula by means of a rotation at finite distance in the complex coordinate plane together with solution of the free-electron wave function's phase and amplitude at finite distance in the complex coordinate plane. The procedure is designed for use with numerically calculated wave functions for many electron atoms and ions. It avoids the use of analytic asymptotic formulas as well as transformation to the dipole acceleration formula and is accurate even for matrix elements between electron states that are close in energy, which is the case for which the alternative integration-by-parts method is inaccurate. We present comparisons of our numerical procedure with both analytic results and results of the integration-by-parts procedure for the case of free-free electron transitions in a pure Coulomb field.

\section{INTRODUCTION}

Radiative transition matrix elements between continuum states of an electron moving in the vicinity of an atom or an ion are central to the study of electron bremsstrahlung $^{1,2}$ and the continuous absorption spectra of stars. ${ }^{3-9}$ These traditional applications plus increasing recent interest in multiphoton absorption processes above the ionization theshold ${ }^{10}$ have led theorists to reexamine numerical techniques for evaluating such free-free radial matrix elements. ${ }^{11-19}$ Among the procedures employed are the use of the acceleration form of the electric dipole operator, ${ }^{4,13,14}$ use of an expansion based on repeated integrations by parts, ${ }^{6,12,16}$ use of the analytic asymptotic expressions for the wave functions beyond some large radius, ${ }^{5,8}$ and use of an analytic Green's function technique based on quantum defect theory. ${ }^{17,18}$ As in the present work, some of these methods employ integration contours in the complex coordinate plane. ${ }^{14,17,18}$

The general nature of the difficulty in evaluating freefree matrix elements numerically is well recognized. Theoretical methods for many-electron systems generally produce wave functions that are most accurate at large distances. ${ }^{4,7}$ The length form of the electric dipole operator, which weights the asymptotic region most heavily, however, leads to radial integrals that are only conditionally convergent. Direct integration along the real axis is unsatisfactory: oscillations of the integrand quickly lead to numerical inaccuracies. The alternative of using analytic asymptotic forms (which permit analytic integration) at finite distances from the origin is also inaccurate.

The ideal procedure when using standard manybody numerical wave functions is to employ the length form of the electric dipole operator from the origin out to the finite distance $r_{0}$ at which exchange integrals between the free electron and the bound electrons are negligible. One would like to employ a procedure that obtains a converged value for the one-electron free-free radial matrix element from $r_{0}$ to $\infty$ without the necessity of numerically integrating oscillating wave functions over a large range in $r$. Rotation of the integration contour into the complex coordinate plane meets this need. In comparison with this ideal procedure, previously employed procedures have limitations. The iterative integration-by-parts procedure ${ }^{6,12,16}$ has convergence difficulties when the energy difference between the initial and final free-electron states becomes small. The use of analytic asymptotic forms ${ }^{5,8}$ for the free-electron wave functions requires numerical integration of oscillating wave functions out to a large radial distance $r_{0}$ if this approximation is to be valid to high accuracy at $r_{0}$ and beyond. The use of quantum defect theory ${ }^{17,18}$ requires either experimental data or a separate theoretical calculation in order to obtain the quantum defect theory parameters that are required. Finally, the transformation of the electric dipole transition operator to the acceleration form ${ }^{4,13,14}$ or to a mixed-gauge representation ${ }^{19}$ is probably not necessary to obtain accurate results when carrying out the radial integrations in the complex coordinate plane.

We show here that highly accurate free-free radial matrix elements may be obtained simply using the dipole length operator and a rotated straight-line trajectory in the complex coordinate plane. For the case of a long-range Coulomb field, we adapt the procedure of Martins ${ }^{20}$ to obtain highly accurate Coulomb phases and amplitudes in the complex plane. Other cases for the asymptotic poten- 
tial seen by the free electron may be treated analogously according to the procedures of Burgess. ${ }^{21}$ We compare our numerical procedure with the commonly used ${ }^{6.12 .16}$ integration-by-parts procedure for the case of free-free transitions in a pure Coulomb field in order to check each of the two methods against analytical predictions.

\section{TWO NUMERICAL METHODS FOR FREE-FREE RADIAL INTEGRALS}

\section{A. Generalities}

The reduction to radial integrals of the free-free transition matrix element between two many-electron, multichannel wave functions has been carried out elsewhere. ${ }^{11,13}$ We focus, therefore, solely on the one-electron radial integral,

$$
\begin{aligned}
\int_{0}^{\infty} u\left(\epsilon_{1} l_{1} ; r\right) \hat{f}(r) u\left(\epsilon_{2} l_{2} ; r\right) d r \\
\quad=\int_{0}^{r_{0}} u\left(\epsilon_{1} l_{1} ; r\right) f(r) u\left(\epsilon_{2} l_{2} ; r\right) d r+I\left(r_{0}, \epsilon_{1} l_{1}, \epsilon_{2} l_{2}\right),
\end{aligned}
$$

where

$$
I\left(r_{0}, \epsilon_{1} l_{1}, \epsilon_{2} l_{2}\right) \equiv \int_{r_{0}}^{\infty} u\left(\epsilon_{1} l_{1} ; r\right) f(r) u\left(\epsilon_{2} l_{2} ; r\right) d r
$$

$\widehat{f}(r)$ is a one-electron radial operator having the coordinate space representation $f(r)$, and $u(\epsilon l ; r)$ is a one-electron radial wave function satisfying the following radial equation in atomic units $(e=\hbar=m=1)$ :

$$
\left(\frac{d^{2}}{d r^{2}}-\frac{c}{r^{2}}+\frac{2 z}{r}-v(r)+k^{2}\right) u(\epsilon l ; r)=0 \quad\left(r \geqslant r_{0}\right) .
$$

It is assumed, following Burgess, ${ }^{21}$ that $v(r)$ comprises all short-range potentials, i.e., $r^{2} v(r) \sim 0$ as $r \rightarrow \infty$; that $c=l(l+1)+\alpha$, where $l$ is the electron's orbital angular momentum and $\alpha / r^{2}$ is a long-range dipole potential; that $\epsilon \equiv k^{2} / 2$; and that $z$ is the effective Coulomb charge seen by the free electron. The general solution of Eq. (2) is

$$
u(\epsilon l ; r)=A[\zeta(r)]^{-1 / 2} \sin [\phi(r)+\delta],
$$

where the amplitude function $\zeta(r)$ satisfies the differential equation

$[\xi(r)]^{2}=\left(k^{2}-\frac{c}{r^{2}}+\frac{2 z}{r}-v(r)\right)+\xi^{1 / 2} \frac{d^{2}}{d r^{2}}\left(\xi^{-1 / 2}\right)$,

and the phase function $\phi(r)$ satisfies

$$
\phi(r)=\int^{r} \zeta(r) d r .
$$

Also, $A$ is a normalization constant and $\delta$ is a phase shift.

The solutions of Eqs. (4) and (5) appropriate for various values of $k, c, z$, and $v(r)$ have been treated by Burgess. ${ }^{21}$ For brevity, we consider here only the common case of an electron moving in a Coulomb field, i.e., $k>0$, $c \equiv l(l+1), z>0$, and $v(r)=0$, which has been analyzed in highly accurate numerical detail by Martins. ${ }^{20}$ For this case the following asymptotic forms apply:

$$
\begin{aligned}
& \phi(r) \underset{r \rightarrow \infty}{\longrightarrow} k r+(z / k) \ln (2 k r)-\frac{1}{2} l \pi+\sigma_{l}+\delta, \\
& \zeta(r) \underset{r \rightarrow \infty}{\longrightarrow} k,
\end{aligned}
$$

where $\sigma_{l} \equiv \arg \Gamma[1+l-i(z / k)]$. We also assume the radial wave functions to be energy normalized (in a.u.), so that

$$
A=(2 / \pi)^{1 / 2} .
$$

Substituting Eqs. (3) and (8) in Eq. (1b), we write the integral whose value we seek as

$$
\begin{aligned}
& I\left(r_{0}, \epsilon_{1} l_{1}, \epsilon_{2} l_{2}\right) \\
& \equiv \frac{2}{\pi} \int_{r_{0}}^{\infty} \frac{f(r) \sin \left[\phi_{1}(r)+\delta_{1}\right] \sin \left[\phi_{2}(r)+\delta_{2}\right] d r}{\left(\zeta_{1} \zeta_{2}\right)^{1 / 2}} .
\end{aligned}
$$

We note that the dependence of $I$ on the particular atom or ion being studied comes solely from the phase shifts $\delta_{1}$ and $\delta_{2}$. This fact may be useful for large-scale tabular computations of free-free matrix elements since trigonometric relations may be used to write $\mathrm{Eq} .(9)$ in the form of a sum of products of a factor containing the phase shifts and an integral over the functions $f(r), \phi(r)$, and $\xi(r)$. These latter functions depend only on the long-range field and are independent of the particular atom or ion being studied. In the rest of this paper we keep $\phi(r)$ and $\delta$ together, inside the integral, for simplicity of notation.

\section{B. Integration-by-parts method}

In this method a convergence factor $e^{-\epsilon r}$ is introduced into the integrand in Eq. (9) and the limit $\epsilon \rightarrow 0$ is taken. Using a standard trigonometric expression to combine the two sine functions, we may rewrite Eq. (9) as

$$
I\left(r_{0}, \epsilon_{1} l_{1}, \epsilon_{2} l_{2}\right) \equiv I^{+}-I^{-},
$$

where

$$
\begin{aligned}
& I^{ \pm} \equiv-\lim _{\epsilon \rightarrow 0} \int_{r_{0}}^{\infty} e^{-\epsilon r} \zeta^{ \pm}(r) g^{ \pm}(r) \cos x^{ \pm}(r) d r, \\
& \zeta^{ \pm}(r) \equiv \xi_{2}(r) \pm \zeta_{1}(r), \\
& g^{ \pm}(r) \equiv f(r)\left[\pi\left(\zeta_{1} \xi_{2}\right)^{1 / 2} \zeta^{ \pm}\right]^{-1}, \\
& x^{ \pm}(r)=\left[\phi_{2}(r)+\delta_{2}\right] \pm\left[\phi_{1}(r)+\delta_{1}\right] .
\end{aligned}
$$

Now, since

$$
\zeta^{ \pm} \cos x^{ \pm} d r=d\left(\sin x^{ \pm}\right)
$$

[due to Eq. (5) and the fact that the phase shifts $\delta$ have radial derivatives equal to zero], we may write

$$
I^{ \pm} \equiv-\lim _{\epsilon \rightarrow 0} \int_{r_{0}}^{\infty} e^{-\epsilon r} g^{ \pm}(r) d\left[\sin \chi^{ \pm}(r)\right]
$$

Repeated integration-by-parts results in the following expansion for Eq. (12):

$$
\begin{aligned}
I^{ \pm} \equiv & \left\{\sum_{n=0}^{\infty}\left[\left(\frac{1}{\xi^{ \pm}} \frac{d}{d r}\right)^{n} g^{ \pm}(r)\right]\right. \\
& \left.\times \sin \left(x^{ \pm}(r)+\frac{n \pi}{2}\right)\right\}_{r=r_{0}} .
\end{aligned}
$$

The first four terms of Eq. (13) have been presented by Aymar and Crance. ${ }^{12}$ The first few terms of the expansion in Eq. (13) have also been used in the calculations of Pindzola and Kelly ${ }^{6}$ (who employ the analytic asymptotic forms for the wave functions in their expressions) and of L'Huillier et al. ${ }^{16}$

\section{Complex coordinate rotation method}

In this method the desired integral, Eq. (9), is again defined by Eq. (10a), where now, however, $I^{ \pm}$is defined as follows: 


$$
I^{ \pm} \equiv-\operatorname{Re} \int_{r_{0}}^{\infty} \zeta^{ \pm}(r) g^{ \pm}(r) \exp \left[i x^{ \pm}(r)\right] d r .
$$

Here Re denotes the real part of the integral and $\zeta^{ \pm}(r)$, $g^{ \pm}(r)$, and $x^{ \pm}(r)$ are defined, as before, by Eqs. (10c)-(10e). In order to make the integral in Eq. (14) converge we make the following coordinate transformation:

$$
r \rightarrow r_{0}+r e^{i \theta} \text {. }
$$

Now

$$
\begin{aligned}
I^{ \pm} \equiv & -\operatorname{Re} \int_{0}^{\infty} \zeta^{ \pm}\left(r_{0}+r \exp i \theta\right) g^{ \pm}\left(r_{0}+r \exp i \theta\right) \\
& \times \exp \left[i x^{ \pm}\left(r_{0}+r \exp i \theta\right)\right] e^{i \theta} d r .
\end{aligned}
$$

If we assume $\epsilon_{2}>\epsilon_{1}$ so that $k_{2}>k_{1}$, then from Eqs. (6) and (10e) we see that $x^{ \pm}(r)>0$ for large $r$, and hence we require $0<\theta<\pi$ in order that $I^{ \pm}$converges. In practice, one achieves the fastest convergence by choosing $\theta=\pi / 2$.

In this method $r_{0}$ must be chosen such that the radial one-electron wave function has the asymptotic form in Eq. (3). The phase shift $\delta$ is then a constant (i.e., coordinate independent). One then solves Eqs. (4) and (5) for the amplitude and phase functions in the complex coordinate plane at each position $r$ along the line defined by $r_{0}+r e^{i \theta}$ for large enough $r$ so that the integral in Eq. (16) converges.

\section{RESULTS AND DISCUSSION}

We compare in Table I the integration-by-parts and the complex coordinate rotation procedures, described in the previous section, for evaluating free-free electric dipole length matrix elements in the case of a free electron moving in a pure Coulomb field. For this case analytic results are also shown in Table $I$. They were obtained as described in the Appendix. In this case the phase shifts $\delta$ are, of course, zero and the radial transition operator $f(r)$ is simply equal to $r$.

In evaluating the desired integral in Eq. (1a), the integration from 0 to $r_{0}$ in Eq. (1a) was carried out along the real axis. In the integration-by-parts method, we evaluated the integral $I$ [cf. Eqs. (1b) and (10a)] using the first four terms of Eq. (13). None of the applications ${ }^{6,12,16}$ of this method has used more than four terms. In the complex coordinate rotation method, we evaluated the integral $I$ [cf. Eqs. (1b) and (10a)] using Eq. (16) with $\theta=\pi / 2$. In both methods we used the procedure of Martins ${ }^{20}$ to evaluate the phase [Eq. (5)] and amplitude [Eq. (4)] functions at finite values of $r$. In using the procedure of Martins ${ }^{20}$ in the complex coordinate plane, we found it more convenient to replace Eq. (4.2) of Ref. (20b) by ${ }^{22}$

$$
\phi_{L}=\phi_{L-1}-i \ln \left[\frac{\left(\eta_{L-1}-i \zeta_{L-1}\right)}{\left[\left(\kappa^{2} L^{2}+1\right) / L^{2}\right]^{1 / 2}}\left(\frac{\zeta_{L}}{\zeta_{L-1}}\right)^{1 / 2}\right]
$$

In the notation used by Martins, ${ }^{20} L$ is the electron's orbital angular momentum, $\phi_{L}$ is the phase function that we denote by $\phi, \zeta_{L}$ is the amplitude function that we denote by $\zeta$, and $\kappa \equiv k / z$.

For $r_{0}=20$ a.u., Table I shows that both the integration-by-parts procedure and the complex coordinate rotation procedure are quite accurate when $\Delta \epsilon \equiv \epsilon_{2}-\epsilon_{1}=0.6$ a.u. When $\Delta \epsilon=0.043$ a.u., however, the integration-byparts procedure fails to give accurate results. While the results we give in Table $I$ are those obtained from the first four terms in the expansion of Eq. (13), we were not able to obtain convergence even when higher terms were included. (In fact, this is why we switched to a complex coordinate rotation method!) When $r_{0}=100$ a.u., the integration-by-parts procedure is able to give accurate results for $\Delta \epsilon=0.043$ a.u., but these results are not as accurate as those of the complex coordinate rotation method, and they require integration over a much larger range in $r$ than is required by the complex coordinate rotation method, which obtains accurate results at $r_{0}=20$ a.u.

We conclude that the complex coordinate rotation

\begin{tabular}{|c|c|c|c|c|c|c|c|}
\hline \multirow[b]{2}{*}{$r_{0}(\text { a.u. })^{a}$} & \multirow[b]{2}{*}{$\epsilon_{1}($ a.u. $)$} & \multirow[b]{2}{*}{$l_{1}$} & \multirow[b]{2}{*}{$\epsilon_{2}$ (a.u.) } & \multirow[b]{2}{*}{$l_{2}$} & \multicolumn{3}{|c|}{$\int_{0}^{\infty} u\left(\epsilon_{1} l_{1} ; r\right) r u\left(\epsilon_{2} l_{2} ; r\right) d r$} \\
\hline & & & & & $\begin{array}{l}\text { Integration- } \\
\text { by-parts } \\
\text { result }^{\mathrm{b}}\end{array}$ & $\begin{array}{l}\text { Complex } \\
\text { coordinate } \\
\text { rotation } \\
\text { result }^{c}\end{array}$ & $\begin{array}{l}\text { Analytic } \\
\text { result }^{\mathrm{d}}\end{array}$ \\
\hline 20 & 0.1 & 0 & 0.7 & 1 & 1.29330 & 1.29930 & 1.29930 \\
\hline 20 & 0.1 & 1 & 0.7 & $\mathbf{0}$ & 0.35687 & 0.35695 & 0.35694 \\
\hline 20 & 0.1 & 1 & 0.7 & 2 & 0.72344 & 0.72339 & 0.72339 \\
\hline 20 & 0.1 & 2 & 0.7 & 1 & 0.08781 & 0.08783 & 0.08782 \\
\hline 20 & 0.016 & 0 & 0.059 & 1 & 113.920 & 111.060 & 111.060 \\
\hline 20 & 0.016 & 1 & 0.059 & 0 & 59.234 & $\mathbf{5 7 . 9 4 3}$ & $\mathbf{5 7 . 9 4 3}$ \\
\hline 20 & 0.016 & $\mathbf{1}$ & 0.059 & 2 & 91.986 & 114.240 & 114.240 \\
\hline 20 & 0.016 & 2 & 0.059 & 1 & 34.836 & 33.414 & 33.413 \\
\hline 100 & 0.016 & 0 & 0.059 & 1 & 111.060 & 111.060 & 111.060 \\
\hline 100 & 0.016 & 1 & 0.059 & 0 & 57.935 & $\mathbf{5 7 . 9 4 4}$ & 57.943 \\
\hline 100 & 0.016 & 1 & 0.059 & 2 & 114.240 & 114.240 & 114.240 \\
\hline 100 & 0.016 & 2 & 0.059 & 1 & 33.405 & 33.414 & 33.413 \\
\hline
\end{tabular}
method presented here is a much more reliable and accurate method than the integration-by-parts procedure. The integration-by-parts procedure must be used with care

TABLE I. Free-free dipole length radial integrals for atomic hydrogen.

a Here $r_{0}$ is chosen to be large enough so that the radial wave functions have the form in Eq. (3).

bee Sec. I B.

' See Sec. I C.

' See Appendix. 
when the energy difference between the free-electron's energies in the free-free matrix element becomes small. In fact, it is not clear to us whether the method is always convergent. Given the high numerical accuracy achieved by the complex coordinate rotation method (cf. Table I), it appears also that there is no need to transform the electric dipole operator to the accleration or mixed-gauge representations.

\section{ACKNOWLEDGMENT}

This work was supported in part by National Science Foundation Grant No. PHY-8601429.

\section{APPENDIX: ANALYTIC EVALUATION OF FREE-FREE DIPOLE LENGTH RADIAL INTEGRALS FOR ATOMIC HYDROGEN}

We seek the matrix element of $r^{n}$ (where $n=1$ for the electric dipole operator in length form) between two continuum radial wave functions,

$$
Q=\int_{0}^{\infty} R_{k^{\prime} l^{\prime}}^{*}(r) r^{\prime \prime} R_{k I}(r) d r
$$

The analytic expression for $R_{k l}(r)$, which has the energynormalized asymptotic form

$$
\begin{aligned}
R_{k l}(r) \sim & \left(\frac{2}{\pi k}\right)^{1 / 2} \frac{1}{r} \\
& \times \sin \left(k r+\frac{Z}{k} \ln (2 k r)-\frac{1}{2} l \pi+\sigma_{l}\right),
\end{aligned}
$$

where $\sigma_{l} \equiv \arg \Gamma(l+1-i Z / k)$, is ${ }^{23}$

$$
R_{k l}(r)=C_{k l} e^{-i k r} F[i(Z / k)+l+1,2 l+2,2 i k r],
$$

where

$$
\begin{aligned}
C_{k l} \equiv & 2\left(\frac{Z}{1-\exp [-2 \pi(Z / k)]}\right)^{1 / 2} \\
& \times \prod_{s=1}^{l}\left[s^{2}+\left(\frac{Z}{k}\right)^{2}\right] \frac{(2 k r)^{l}}{(2 l+1) !}
\end{aligned}
$$

and where $F$ is a confluent hypergeometric function. ${ }^{24}$ Substituting Eq. (A3) into Eq. (A1) gives

$$
Q=C_{k^{\prime} l^{\prime}} C_{k l} J_{\gamma}^{s p}\left(\alpha^{\prime}, \alpha\right),
$$

where
$J_{\gamma}^{s p}\left(\alpha^{\prime}, \alpha\right) \equiv \int_{0}^{\infty} e^{-\left(\kappa^{\prime}+\kappa i r / 2\right.} r^{\gamma-1+s} F\left(\alpha^{\prime}, \gamma, \kappa^{\prime} r\right) F(\alpha, \gamma-p, \kappa r) d r$

and the new symbols have the following meanings: $p \equiv 2\left(l^{\prime}-l\right), \quad s \equiv n-\left(l^{\prime}-l\right)+1, \quad \gamma \equiv 2 l^{\prime}+2, \quad \alpha^{\prime} \equiv i Z / k^{\prime}$ $+l^{\prime}+1, \alpha \equiv i Z / k+l+1, \kappa^{\prime} \equiv 2 i k^{\prime}$, and $\kappa=2 i k$. Note that since the radial function $R_{k l}(r)$ defined by $\mathrm{Eq}$. (A3) is real, the complex conjugate in Eq. (A1) has no effect, i.e., we could just as well have chosen to define $\alpha^{\prime}$ and $\kappa^{\prime}$ in Eq. (A5) with $i$ replaced by $-i$.

Equation (A5) is identical in form to Eq. (f.12) of Landau and Lifshitz. ${ }^{24}$ It is evaluated numerically by using the recursion relations of Eqs. (f.15) and (f.16) of Ref. 24 to reduce $J_{\gamma}^{s p}$ to a sum of integrals having $s=p=0$. These integrals $J_{\gamma}^{00}$ are expressed analytically in terms of a hypergeometric function in Eq. (f.12) of Ref. 24.

\section{REFERENCES}

1. A. Sommerfeld, Ann. Phys. (Leipzig) 11, 257 (1931).

2. R. H. Pratt, in Fundamental Processes in Energetic Atomic Collisions, edited by H. O. Lutz, J. S. Briggs, and H. Kleinpoppen (Plenum, New York, 1983), pp. 145-182.

3. A. Pannekoek, Mon. Not. R. Astron. Soc. 91, 166 (1931).

4. G. Peach, Mon. Not. R. Astron. Soc. 130, 361 (1965)

5. J. L. Stilley and J. Callaway, Astrophys. J. 160, 245 (1970).

6. M. S. Pindzola and H. P. Kelly, Phys. Rev. A 14, 204 (1976).

7. M. J. Conneely and S. Geltman, J. Phys. B 14, 4847 (1981).

8. K. L. Bell, K. A. Berrington, and J. P. Croskery, J. Phys. B 15, 977 (1982).

9. K. L. Bell and K. A. Berrington, J. Phys. B 20, 801 (1987).

10. P. Agostini, F. Fabre, and G. Petite, in Mulitphoton Ionization of Atoms, edited by S. L. Chin and P. Lambropoulos (Academic, Orlando, FL, 1984), pp. 133-154.

11. K. L. Bell, P. G. Burke, and A. E. Kingston, J. Phys. B 10, 3117 (1977)

12. (a) M. Aymar and M. Crance, J. Phys. B 14, 3585 (1981); (b) 13, L287 (1980).

13. M. J. Seaton, J. Phys. B 14, 3827 (1981).

14. N. C. Sil, M. A. Crees, and M. J. Seaton, J. Phys. B 17, 1 (1984)

15. M. Gavrila, A. Maquet, and V. Véniard, Phys. Rev. A 32, 2537 (1985)

16. A. L'Huillier, L. Jönsson, and G. Wendin, Phys. Rev. A 33, $3938(1986)$.

17. M. Edwards, $X$. Tang, P. Lambropoulis, and R. Shakeshaft, Phys. Rev. A 33, 4444 (1986).

18. M. Edwards, X. Tang, and R. Shakeshaft, Phys. Rev. A 35, 3758 (1987).

19. C. Chang and E. J. Robinson, J. Phys. B 20, 963 (1987).

20. (a) P. de A. P. Martins, J. Phys. B 1, 154 (1968); (b) Proc. Cambridge Philos. Soc 69, $167(1971)$

21. A. Burgess, Proc. Phys. Soc. London 81, 442 (1963).

22. Equation (17) is obtained first, by using Eqs. (1.3) and (4.1) of Ref. (20b) to replace $\left(\ln \zeta_{L-1}\right)^{\prime}$ in Eq. 2.2 of Ref. $20(a)$ and, second, by taking the natural $\log$ of the result.

23. H. A. Bethe and E. E. Salpeter, Quantum Mechanics of One- and Two-Electron Atoms (Springer, Berlin, 1957), p. 24.

24. L. D. Landau and E. M. Lifshitz, Quantum Mechanics (Addison-Wesley, Reading, MA, 1965), 2nd ed., Mathematical Appendices, Sec. (d)-Sec. (f), pp. $600-610$. 